\title{
Harnessing plant viruses to treat autoimmune diseases
}

The induction of immune tolerance is a promising approach for treating autoimmune diseases. Several strategies have been trialled to induce immune tolerance, including the use of antigen-specific peptide vaccines, which have had some success but can trigger unwanted immune responses owing to the use of an adjuvant. The results of a new study suggest that plant viruses could be harnessed to deliver specific peptides without the need for an adjuvant, thereby improving the efficacy of peptide vaccines.

Nanoparticles created from plant virus coat protein subunits can be genetically engineered to express an antigen-specific peptide related to an autoimmune disease. These nanoparticles can then be grown in their natural plant hosts (a technique known as molecular farming) before being collected for use.
"We started working 20 years ago on molecular farming as an enabling technology to solve the challenges posed by autoimmune diseases, in particular those related to tolerance induction," says corresponding author Linda Avesani. "In our new paper, we demonstrated for the first time that bio-designed nanomaterials can be used to prevent autoimmune diabetes and to treat autoimmune arthritis."

Avesani and colleagues created nanoparticles from tomato bushy stunt virus (TBSV) that expressed one of two peptides (pLIP1 or pFADK2), which had previously been identified in a peptide library screen as being immunodominant in patients with seronegative rheumatoid arthritis. Administration of these nanoparticles to mice with collagen-induced arthritis reduced their symptoms (and in the case of pLIP1, abolished them completely) compared with treatment with the same peptide and an adjuvant or with saline, and produced similar or better results to treatment with the glucocorticoid dexamethasone.

In addition, the researchers tested wild-type TBSV on its own, which was also able to reduce the symptoms of arthritis, albeit to a lesser degree than the peptide-engineered nanoparticles, suggesting that TBSV has innate immunomodulatory properties. "These results indicate that the virus structure acts both as a carrier (stabilizing the peptide) and as an adjuvant," explains Avesani.

Similar results were also achieved in a model of autoimmune diabetes using a different plant virus. Avesani and colleagues hope to expand their use of this technique to other autoimmune diseases and to develop the nanoparticles for use in humans.

Joanna Clarke

ORIGINAL ARTICLE Zampieri, R. et al. Prevention and treatment of autoimmune diseases with plant virus nanoparticles. Sci. Adv. 6, eaaz0295 (2020)

\section{Uncovering the pro-resolving gene network in RA}

Resolution of inflammation is important for restoring tissue homeostasis, and failure to resolve can lead to chronic inflammatory diseases such as rheumatoid arthritis (RA). New findings shed light on this dynamic process, including the identification of three previously unknown pro-resolving factors, which could guide the development of new therapies and biomarkers for predicting disease remission.

“A variety of cellular processes contribute to anti-inflammatory responses," explains Wan-Uk Kim, corresponding author on the new study. "Despite advancements in our understanding of inflammation resolution, global analyses have not been sufficiently explored to systematically discover the factors or pathways underlying resolution of chronic inflammatory diseases."

To address this issue, Kim and colleagues used a systems biology approach to characterize the temporal changes in synovial gene expression profiles of mice with collagen-induced arthritis (CIA), including during the induction, peak and resolution phases of disease. Network analysis of differentially expressed genes across these phases identified three genes associated with spontaneous resolution of CIA: Itgb1, Rps3 and Ywhaz.

All three genes encoded secretory proteins that could suppress the production of pro-inflammatory cytokines (such as TNF and IL-6) by a variety of effector cells in vitro, including by macrophages and fibroblast-like synoviocytes. Notably, levels of YWHAZ were upregulated in the sera of mice during the resolution phase, suggesting that this pro-resolving factor could serve as a clinical biomarker. Indeed, in patients with RA, urinary levels of YWHAZ were increased following 4-6 months

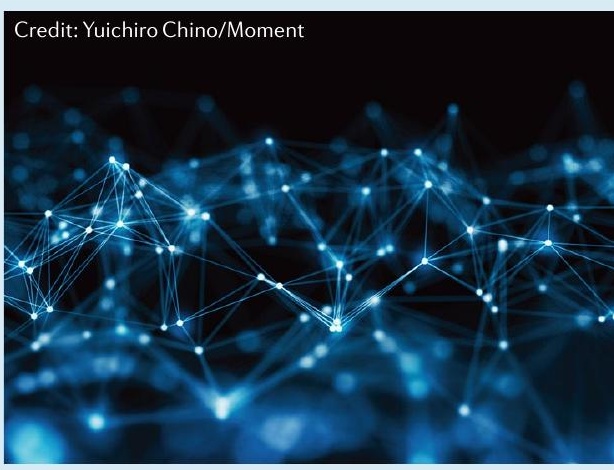

treatment with anti-rheumatic drugs, but only in those patients who had responded well to treatment and not in patients with a moderate or no response.

YWHAZ also showed potential as a therapeutic target. In mice with $\mathrm{CIA}$, intra-articular injection of an Ywhazcontaining adenovirus suppressed progression of disease, including synovial inflammation, joint destruction and levels of pro-inflammatory cytokines. Jessica McHugh progression of disease

ORIGINAL ARTICLE Kong, J.-S. et al. Dynamic transcriptome analysis unveils key pro-resolving factors of chronic inflammatory arthritis. J. Clin. Invest. https://doi.org/10.1172/JCI126866 (2020) 